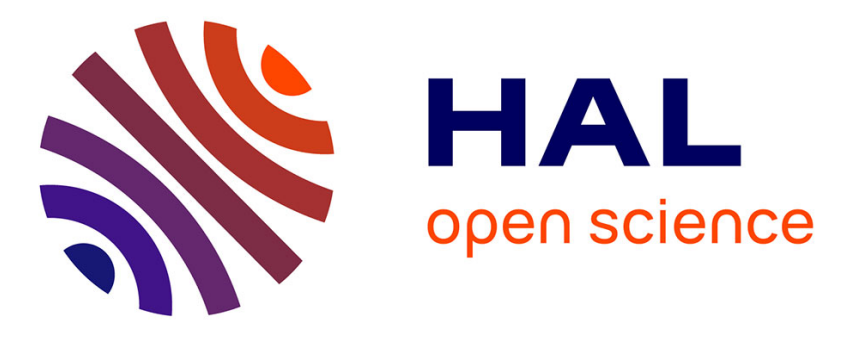

\title{
Control of the pore texture in nanoporous silicon via chemical dissolution
}

\author{
E. Secret, C. C. Wu, A. Chaix, A. Galarneau, P. Gonzales, D. Cot, M. J. \\ Sailor, J. Jestin, J. M. Zanotti, F. Cunin, et al.
}

\section{- To cite this version:}

E. Secret, C. C. Wu, A. Chaix, A. Galarneau, P. Gonzales, et al.. Control of the pore texture in nanoporous silicon via chemical dissolution. Langmuir, 2015, 31, pp.8121-8128. 10.1021/acs.langmuir.5b01518 . hal-01227191

\section{HAL Id: hal-01227191 \\ https://hal.science/hal-01227191}

Submitted on 4 Feb 2021

HAL is a multi-disciplinary open access archive for the deposit and dissemination of scientific research documents, whether they are published or not. The documents may come from teaching and research institutions in France or abroad, or from public or private research centers.
L'archive ouverte pluridisciplinaire HAL, est destinée au dépôt et à la diffusion de documents scientifiques de niveau recherche, publiés ou non, émanant des établissements d'enseignement et de recherche français ou étrangers, des laboratoires publics ou privés. 


\section{TOC Figure}

\section{Control of the Pore Texture in Nanoporous Silicon via Chemical Dissolution}

Emilie Secret, ${ }^{1}$ Chia-Chen Wu, ${ }^{2}$ Arnaud Chaix, ${ }^{1}$ Anne Galarneau, ${ }^{1}$ Philippe Gonzalez, ${ }^{1}$ Didier

Cot, ${ }^{3}$ Michael J. Sailor, ${ }^{2}$ Jacques Jestin, ${ }^{4}$ Jean-Marc Zanotti, ${ }^{4}$ Frédérique Cunin, ${ }^{1}$ Benoit Coasne $e^{1,5,6^{*}}$
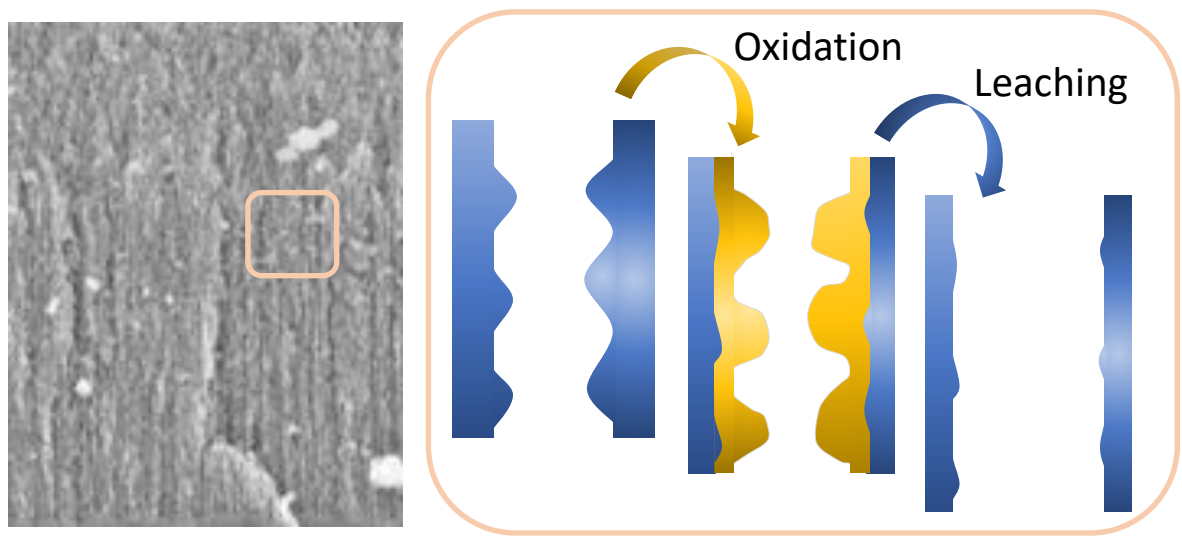


\section{Control of the Pore Texture in Nanoporous Silicon via Chemical Dissolution}

Emilie Secret, ${ }^{1}$ Chia-Chen Wu, ${ }^{2}$ Arnaud Chaix, ${ }^{1}$ Anne Galarneau, ${ }^{1}$ Philippe Gonzalez, ${ }^{1}$ Didier Cot ${ }^{3}$ Michael J. Sailor, ${ }^{2}$ Jacques Jestin, ${ }^{4}$ Jean-Marc Zanotti, ${ }^{4}$ Frédérique Cunin, ${ }^{1}$ Benoit Coasne $e^{1,5,6^{*}}$

1 Institut Charles Gerhardt Montpellier, UMR 5253 CNRS/ENSCM/UM2/UM1, 8 rue de l'Ecole Normale, 34296 Montpellier, France.

${ }^{2}$ Department of Chemistry and Biochemistry, University of California San Diego, La Jolla, CA 92093-0358, USA.

${ }^{3}$ Institut Européen des Membranes, ENSCM/UM2/UMR 5635, Place Eugène Bataillon CC 047, 34095 Montpellier, France.

${ }^{4}$ Laboratoire Leon Brillouin, CEA-Saclay, 91191 Gif sur Yvette Cedex, France.

${ }^{5}$ MultiScale Materials Science for Energy and Environment, CNRS/MIT (UMI 3466), 77 Massachusetts Avenue, Cambridge, MA 02139, USA.

${ }^{6}$ Department of Civil and Environmental Engineering, Massachusetts Institute of Technology, 77 Massachusetts Avenue, Cambridge, MA 02139, USA.

* To whom correspondence should be addressed. E-mail: coasne@mit.edu 


\begin{abstract}
The surface and textural properties of porous silicon (pSi) control many of its physical properties essential to its performance in key applications such as optoelectronics, energy storage, luminescence, sensing, and drug delivery. Here, we combine experimental and theoretical tools to demonstrate that the surface roughness at the nanometer scale of pSi can be tuned in a controlled fashion using partial thermal oxidation followed by removal of the resulting silicon oxide layer with hydrofluoric acid (HF) solution. Such a process is shown to smooth the pSi surface by means of nitrogen adsorption, electron microscopy, and small angle X-ray or neutron scattering. Statistical Mechanics Monte Carlo simulations, which are consistent with the experimental data, support the interpretation that the pore surface is initially rough and that the oxidation/oxide removal procedure diminishes the surface roughness while increasing the pore diameter. As a specific example considered in this work, the initial roughness $\xi \sim 3.2 \mathrm{~nm}$ of pSi pores having a diameter of $7.6 \mathrm{~nm}$ can be decreased down to 1.0 $\mathrm{nm}$ following the simple procedure above. This study allows envisioning the design of $\mathrm{pSi}$ samples with optimal surface properties towards a specific process.
\end{abstract}




\section{Introduction}

The internal nanostructure of porous silicon $(\mathrm{pSi})$ is determinant when applied to fields such as optoelectronics, energy storage, luminescence imaging, sensing and drug delivery. ${ }^{1-4}$ Most of these applications rely on the large specific surface area of $\mathrm{pSi}$. While pore diameter and porous volume are important parameters to control when designing pSi-based sensors or controlled drug delivery systems, pore walls features (thickness, homogeneity, nanocrystallite diameter) also rule pSi optical performances. ${ }^{4}$ Obtained by electrochemical etching of doped crystalline silicon wafers, the relative simplicity by which the pore dimensions of $\mathrm{pSi}$ can be controlled also provides an ideal model system to study classical theories of adsorption and capillary condensation in nanoporous materials. ${ }^{5-8}$ As a result, porous silicon was used to investigate important classical theories such as Cohan's conjecture, which suggests that condensation should be irreversible for pores opened at both end and reversible for pores closed at one end. ${ }^{9}$

Along with its intrinsic chemical and physical properties (including optical/electrical, and adsorbing/confining properties), $\mathrm{pSi}$ presents some intrinsic roughness which has been speculated for a long time and recently evidenced ${ }^{7}$ but remains essentially unexplored. The lack of a detailed understanding of the textural and surface properties of columnar pSi represents an obstacle for its optimal use in the practical applications mentioned above. Furthermore, the nonnegligible, uncontrolled surface roughness of $\mathrm{pSi}$ also represents a challenge for its use as an ideal material for testing theories on adsorption and transport upon confinement. Such surface roughness can be present at different scales, typically from atomistic surface roughness $(<1$ $\mathrm{nm}$ ), nanoscale disorder (length scale $\sim 1 \mathrm{~nm}$ to $10 \mathrm{~nm}$ ), and even at mesoscopic $(>10 \mathrm{~nm})$ or macroscopic scales $(1 \mu \mathrm{m})$. The disorder length scale can be probed using diffraction techniques such as small angle X-ray or neutron scattering which allow determining the Porod range and 
its corresponding exponent ( $\neq 4$ for a rough surface). In the context of the present paper, surface roughness refers to nanometer scale disorder which significantly affects the adsorption properties of $\mathrm{pSi}$. As an illustration of the important nanoscale surface disorder in $\mathrm{pSi}$, adsorption in this material in which the pores are open at both ends or open at only one end violates Cohan's conjecture, exhibiting pronounced hysteresis loops. ${ }^{5}$ While some authors attribute such hysteresis loops to surface roughness of the pores along their axis, ${ }^{7}$ other authors have argued that pores in pSi do not exhibit sufficient pore disorder to explain irreversibility of capillary condensation in closed pores. ${ }^{10}$ Surface disorder can also be invoked to explain the large discrepancies, by a factor $\sim 2$, observed between the pore size distribution of porous silicon, as estimated from scanning electron microscopy (SEM), and that estimated on the basis of the Kelvin equation known to be accurate for large pores as in columnar $\mathrm{pSi}^{5,11}$ (typically for pores larger than $15 \sigma$ where $\sigma$ is the size of the adsorbate molecule, $\sigma \sim 0.36 \mathrm{~nm}$ for $\mathrm{N}_{2}$ ). ${ }^{12}$

Here, we report a joint experimental and theoretical study of the surface and nanotextural properties of columnar pSi for different pSi materials. Starting from as-synthesized pSi, the effect of surface roughness was probed using a chemical treatment that selectively removes the finer features in $\mathrm{pSi}$. The pore wall smoothing process involves controlled thermal oxidation followed by removal of the resulting silicon oxide layer with hydrofluoric acid (HF) solution. Partial thermal oxidation combined with wet etching procedure has been used previously for enlarging macropores and smoothing surface heterogeneities. ${ }^{13-15}$ Other method, such as sintering, have also been shown to reduce the pore surface roughness. ${ }^{16-18}$ Here, significant changes in the texture of the pSi sample were observed at each step of this treatment: (1) freshly prepared $\mathrm{pSi}$, (2) oxidized $\mathrm{pSi}$, and (3) oxidized pSi after immersion into HF. The porous volume and specific surface area of the sample were monitored by low temperature nitrogen 
$\left(\mathrm{N}_{2}\right)$ adsorption at each step while the surface roughness was assessed using neutron or X-ray scattering. Secondly, we report a phenomenological model combined with Grand Canonical Monte Carlo simulations to establish the relationship between the surface roughness and adsorption properties of $\mathrm{pSi}$ along the three stages of the pore smoothing treatment described above. The simulated adsorption isotherms, which qualitatively verify their experimental counterpart, support the interpretation that the pore surface is initially rough on the nanometer scale and that the oxidation/oxide removal treatment diminishes surface roughness while increasing pore diameter. The link established for the first time between surface roughness and adsorption properties together with the smoothing procedure paves the way for the engineering of pSi samples with optimal properties.

\section{Experimental methods and materials}

\subsection{Preparation of porous silicon samples}

Freshly etched $\mathrm{pSi}$ samples were prepared from single-crystalline $\mathrm{p}^{++}$-type $\mathrm{Si}$ (boron doped, 0.8-1.2 $\mathrm{m} \Omega . \mathrm{cm}$ resistivity, $<100>$ orientation, Siltronix Inc.) by electrochemical etching in a 3:1 volume ratio solution of aqueous hydrofluoric acid (48\%, Sigma-Aldrich)/ethanol. Removal of the parasitic layer was ensured by first applying a current density of $75 \mathrm{~mA} . \mathrm{cm}^{-2}$ for 30 seconds to the wafer prior to etching, followed by dissolution of the thin pSi film in a $1 \mathrm{M} \mathrm{NaOH}$ solution for two minutes, and followed by rinsing with a 1:1:3 volume ratio $\mathrm{HF}: \mathrm{H}_{2} \mathrm{O}$ :Ethanol solution. The samples were then rinsed three times with ethanol and dried under a stream of nitrogen. Etching was then performed in a teflon cell with a platinum ring counter electrode. Two different etching conditions were applied: A constant current density of $15 \mathrm{~mA} . \mathrm{cm}^{-2}$ was applied for 30 minutes, or a constant current density of $75 \mathrm{~mA} . \mathrm{cm}^{-2}$ was applied for 5 minutes. The samples were then rinsed 3 times with ethanol and dried under a stream of nitrogen. After 
etching, the samples were oxidized at $400^{\circ} \mathrm{C}$ for 1 hour and cooled down to room temperature in order to generate a layer of silicon oxide at the pSi surface. These oxidized samples were then immersed in a 1:1 volume ratio solution of hydrofluoric acid/ethanol for 5 seconds, rinsed 3 times with ethanol and rinsed 3 times with pentane. This procedure allowed dissolution of the silicon oxide layer formed upon thermal oxidation and recovery of silicon hydride terminated surfaces

\subsection{SEM and $\mathrm{N}_{2}$ adsorption/desorption analysis}

At each step of the preparation, the samples were analyzed by means of nitrogen $\left(\mathrm{N}_{2}\right)$ adsorption and Scanning Electron Microscopy (SEM), in order to determine the surface area, porous volume and diameter of the pores for each sample. SEM images were obtained with a high resolution Field Emission Gun S-4800 Hitachi microscope. Prior to the SEM analysis, the pSi samples were metalized with a thin layer of gold in order to avoid sample charging anomalies. Nitrogen adsorption isotherms were recorded at $77 \mathrm{~K}$ using a micromeritics ASAP 2020 volumetric apparatus. Prior to the adsorption experiment, the samples were outgassed overnight in situ at $303 \mathrm{~K}$. The specific surface area of a given sample is measured using the BrunauerEmmett-Teller (BET) technique. This method allows estimating specific surfaces from adsorption isotherm measurements on the basis of a statistical mechanics model developed to describe multilayer adsorption. The hypothesis of the BET model is that $(i)$ adsorption occurs on homogeneous sites with an interaction energy $\varepsilon_{1}$ (the surface density of sites is constant) and (2) molecules can adsorb on each other. In the latter case, the adsorption energy $\varepsilon_{0}$ for molecules adsorbed on top of the first adsorbed layer is constant. With these assumptions, the adsorbed amount $N$ is related to the relative pressure $\mathrm{P} / \mathrm{P}_{0}$ through the surface density of sites $N_{0}$ and a dimensionless energy parameter $\mathrm{C}$ related to $\varepsilon_{0}$ and $\varepsilon_{1}$. By multiplying $N_{0}$ by the molecular surface area of $0.162 \mathrm{~nm}^{2}$ (for an adsorbed nitrogen molecule), one can estimate the specific 
surface area of a given sample. The pore sizes were determined using the BdB (Broekhoff-de Boer) method from the nitrogen adsorption branch. ${ }^{19}$ The latter allows estimating pore size distribution by correcting Kelvin equation for the interaction with the pore surface. In this model, the pores are assumed to be independent cylinders with pore filling occurring at a pressure given by Laplace pressure. The porous volume was measured from the maximum adsorbed amount measured at pressures above capillary condensation. Assuming that the density of the confined liquid equal that of bulk liquid nitrogen, one can convert an adsorbed amount at saturation into a porous volume.

\subsection{Small Angle Neutron Scattering and Small Angle X-ray Scattering}

To probe the pore surface roughness and the thickness of the oxide layer, small angle neutron scattering (SANS) and small angle X-ray scattering (SAXS) were performed on all the samples. The (SANS) measurements were performed at the Laboratoire Léon Brillouin (LLB, SaclayFrance) on the spectrometer PAXY, at fixed wavelength $\lambda=6 \AA$ and by varying the sample-todetector distance D from $1 \mathrm{~m}$ to $6.7 \mathrm{~m}$ to cover a total Q-range from $10^{-2}$ to $4 \times 10^{-1} \AA^{-1}$. Samples were measured in calibrated glass cells. The standard treatment of the resulting 2D patterns was done with the software Pasinet. These $2 \mathrm{D}$ data were reduced to $1 \mathrm{D}$ spectra $\mathrm{I}(\mathrm{Q})$ after radial averaging around the center of scattering. Standard corrections by sample thickness, neutron beam transmission, empty cell signal subtraction, detector efficiency, subtraction of incoherent scattering were applied to get the scattered intensities on absolute scale $\left(\mathrm{cm}^{-1}\right)$.

The (SAXS) measurements were performed at the Institut Français du Pétrole Energies Nouvelles (IFPEN, Rueil-Malmaison-France) on a copper rotating anode at fixed wavelength of $\lambda=1.5418 \AA$. We used a 2D detector with two sample to detector distances of $1.5 \mathrm{~m}$ and 
$0.65 \mathrm{~m}$ that enable having access to a full $\mathrm{Q}$ range from $8.10^{-3}$ to $0.6 \AA^{-1}$. The scattered intensities were normalized by the sample thickness, the measurement time and the sample transmission. Calibrations were made using references, toluene for calibrating the intensity and silver behenate for calibrating the Q values.

\section{Results and discussion}

\subsection{Experimental study of the textural properties of pSi}

\subsection{1. $\mathrm{N}_{2}$ adsorption}

Two pSi films with distinct pore diameters were prepared by etching highly doped $\mathrm{p}$ type silicon at a current density of $15 \mathrm{~mA} \cdot \mathrm{cm}^{-2}$ for $30 \mathrm{~min}$, and at a current density of $75 \mathrm{~mA} \cdot \mathrm{cm}^{-2}$ for $5 \mathrm{~min}$, respectively. For both $\mathrm{pSi}$ films, the Si wafers underwent a pre-cleaning treatment, which allows inhibiting the formation of a parasitic layer with obstructed porosity that could affect the adsorption properties of the pSi films. The effect of the parasitic layer on the adsorption properties of $\mathrm{pSi}$ films is presented in detail in the supporting information file (Figure S1 and Table S1). The pSi films were then exposed to different chemical treatments (oxidation at $400^{\circ} \mathrm{C}$ for one hour and then $\mathrm{HF} / \mathrm{ethanol}$ treatment). The texture of the pSi films was investigated for the three different forms of the porous film: the freshly etched pSi film, the same pSi film oxidized at $400^{\circ} \mathrm{C}$, and the same oxidized pSi film after immersion in a HF/ethanol solution. Each sample was then analyzed by means of SEM and $\mathrm{N}_{2}$ adsorption. In the following, only results from the series etched at $15 \mathrm{~mA} \cdot \mathrm{cm}^{-2}$ for $30 \mathrm{~min}$ are presented. The results from the samples etched at $75 \mathrm{~mA} \cdot \mathrm{cm}^{-2}$ for $5 \mathrm{~min}$ led to the same conclusions (Supplementary Information, Figure S2-S3 and Table S2). The SEM plane and cross-section images are presented in Figure 1 for the freshly etched pSi film, the pSi film after oxidation, 
and the pSi film after immersion in HF. No obvious difference was observed on the plane-view images of the three pSi samples (Figure 1). In particular, the number of pores seems to remain constant, which suggests that the structure remains intact upon oxidation and removal of the silicon oxide layer in HF. Moreover, we note that even a partial collapse of the pore walls in pSi upon oxidation/removal of the oxide layer would lead to a drastic change in the $\mathrm{N}_{2}$ adsorption isotherms and/or X-ray/neutron small angle scattering study (presented below); in contrast, both the $\mathrm{N}_{2}$ adsorption and scattering data show that the morphology of the samples remain identical along the three stages: freshly etched $\mathrm{pSi}$, oxidized $\mathrm{pSi}$, and oxidized sample dipped in HF. 

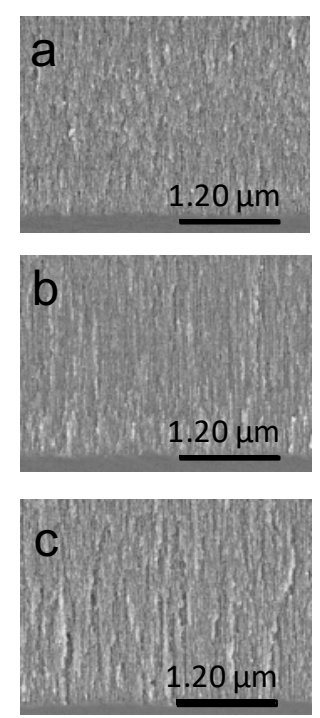
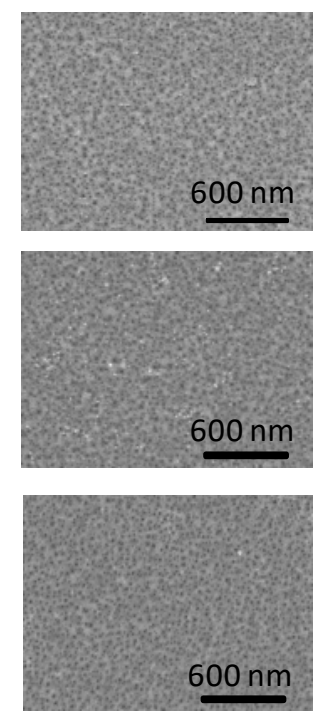

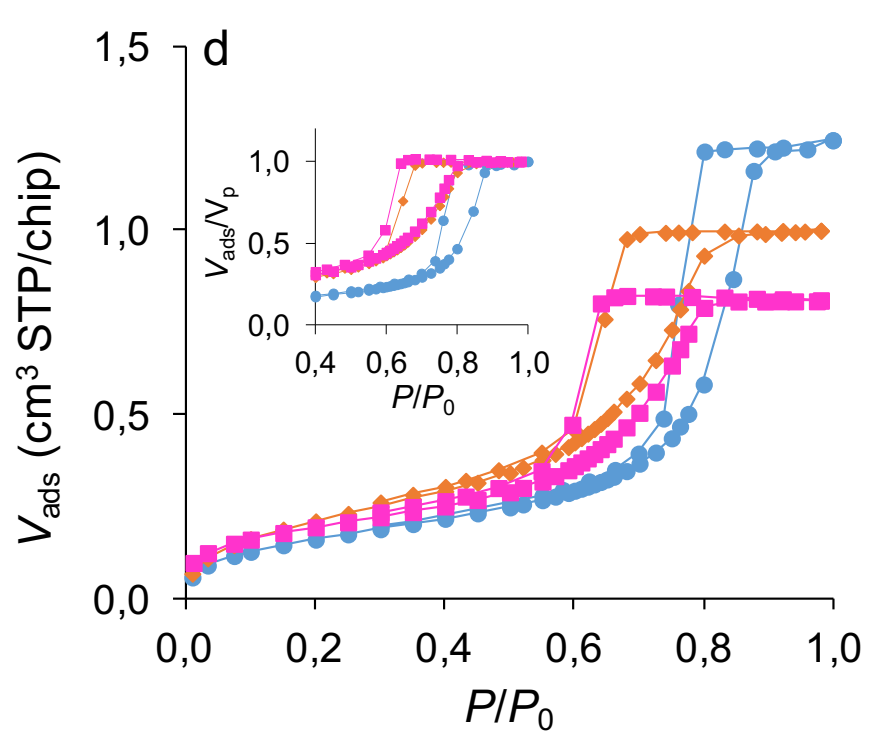

Figure 1. SEM images and $\mathrm{N}_{2}$ adsorption isotherms at $77 \mathrm{~K}$ for pSi samples after the different chemical treatments. Cross-sectional (left) and plan-view (right) SEM images of pSi sample etched at $15 \mathrm{~mA} . \mathrm{cm}^{-2}$ for $30 \mathrm{~min}$ : freshly etched pSi film (a), same pSi film oxidized at $400^{\circ} \mathrm{C}$ for $1 \mathrm{~h}$ (b), same oxidized pSi film after immersion in a HF/ethanol solution (c). The cross-section images, which show the section of the pores, are close-up views of the bottom of the pSi layer. These high magnification images do not display the top surface opened towards the external environment. (d) Nitrogen adsorption isotherms at $77 \mathrm{~K}$ for pSi etched at $15 \mathrm{~mA} . \mathrm{cm}^{-2}$ for $30 \mathrm{~min}$ : (४) freshly etched pSi sample, (घ) pSi sample oxidized at $400^{\circ} \mathrm{C}$ for $1 \mathrm{~h},(\bullet) \mathrm{pSi}$ sample oxidized at $400^{\circ} \mathrm{C}$ for $1 \mathrm{~h}$ and immersed in a HF/ethanol solution for $5 \mathrm{~s}$. The inset shows the same data normalized to the maximum adsorbed volume for each sample $\left(V_{\text {ads }} / V_{\mathrm{p}}\right)$.

The $\mathrm{N}_{2}$ adsorption isotherms at $77 \mathrm{~K}$ for the three forms of the pSi film etched at $15 \mathrm{~mA} . \mathrm{cm}^{-2}$ for 30 min are shown in Figure 1. Table 1 summarizes the values of the mean diameter, the porous volume and the specific surface area estimated from the $\mathrm{N}_{2}$ adsorption isotherms. We 
also show in the supporting information file the pore size distribution as estimated from the Density Functional Theory (Figure S4). Adsorption-related quantities including specific surface areas and porous volumes are expressed per chip of pSi throughout this manuscript. A chip corresponds to the porous film formed in an initial silicon wafer of a geometrical surface of 3.6 $\mathrm{cm}^{2}$. This implies that all steps were performed with the same amount of porous material so that data obtained after the different surface chemical treatments can be compared without any conversion. The freshly etched pSi film exhibits a mean pore diameter of $7.6 \mathrm{~nm}$, a porous volume of $4.4 \times 10^{-3} \mathrm{~mL} / \mathrm{chip}$, and a specific surface area of $2.34 \mathrm{~m}^{2} /$ chip. As usually observed for columnar $\mathrm{pSi}$, the pore size distribution inferred from $\mathrm{N}_{2}$ adsorption drastically underestimates $(\sim 50 \%)$ that observed in electron microscopy images. The value for the $\mathrm{C}_{\mathrm{BET}}$ parameter of 31 for the freshly etched pSi film is consistent with the weakly interacting surface of pSi due to the presence of silicon hydride surface groups. After oxidation, the mean diameter of the pores decreased from $7.6 \mathrm{~nm}$ to $7.2 \mathrm{~nm}$. The porous volume and the specific surface decreased to $3.6 .10^{-3} \mathrm{~mL} / \mathrm{chip}$ and $2.01 \mathrm{~m}^{2} /$ chip, respectively. In this case, upon oxidation, insertion of oxygen atoms in the silicon matrix provokes the swelling of the pSi walls. ${ }^{20,21}$ Such a swelling induces a decrease of the pore diameter and a decrease of the porous volume and surface area. The $\mathrm{C}_{\mathrm{BET}}$ parameter for the oxidized sample $\mathrm{C}_{\mathrm{BET}} \sim 75$, which is much larger than for the freshly etched sample, is consistent with what is usually obtained for silicon oxide surfaces. ${ }^{12}$ After immersion of the oxidized pSi film in HF, a large increase of the mean pore diameter from 7 to $11 \mathrm{~nm}$ is observed, as the relative pressures of adsorption and desorption are shifted to higher values (Figure 1d, blue circles). This final value for the mean pore diameter of the pSi film $(11 \mathrm{~nm})$ is higher than the initial pore diameter of the freshly etched pSi film (7.6 $\mathrm{nm})$. The increase in the mean pore diameter for the $\mathrm{pSi}$ film after removal of the silicon oxide from the matrix walls is logically accompanied with an increase of the porous volume up to 5.4.10 $10^{-3} \mathrm{~mL} / \mathrm{chip}$. It is due to the dissolution and removal of the silicon oxide present in the pSi 
walls upon exposure to HF. Surprisingly, the specific surface area of the pSi film significantly decreases down to $1.73 \mathrm{~m}^{2} /$ chip after the oxide dissolution step. Indeed, in the case of pores with smooth wall surface, an increase of the porous volume would necessarily imply an increase of the pores perimeter and, hence, an increase of their surface. Consequently, a decrease of the specific surface of the sample is a priori not compatible with a concomitant increase of the porous volume for pores with smooth wall surface. Considering that the number of pores remains constant over the successive surface chemical treatments and that their shape remains columnar (Figure 1a, b, c), the results from the $\mathrm{N}_{2}$ adsorption analysis suggest that the pores in freshly etched porous silicon exhibits significant surface roughness and textural disorder which are smeared out upon oxidation followed by etching in HF. This interpretation is consistent with previous conclusion drawn by Naumov et al. who attributed the triangular shape of the capillary hysteresis loops observed for pSi to important surface disorder. ${ }^{7}$ In particular, our results suggest that surface oxidation is preferential in regions where the surface is convex, i.e. on bumps or constrictions as schematically sketched in Figure 2. Indeed, it was observed that the desorption branch for the oxidized pSi film is always shifted towards lower relative pressure to a larger extent than the adsorption branch, resulting in an enlargement of the hysteresis loops (insert of Figure 1d). This observation also supports the idea that reactivity to oxidation of convex regions and constrictions in the silicon walls, which exhibit large specific surface area and low curvature radius, is higher than that of smooth regions. 
Table 1: Textural parameters estimated from the $\mathrm{N}_{2}$ adsorption measurements for the $\mathrm{pSi}$ films etched at $15 \mathrm{~mA} \cdot \mathrm{cm}^{-2}$ for $30 \mathrm{~min} .<\mathrm{D}>$ denotes the mean pore diameter, $\mathrm{D}_{\mathrm{O}}$ is the pore opening. $V_{\mathrm{p}}$ is the porous volume as measured from the maximum adsorbed amount. $\mathrm{C}_{\mathrm{BET}}$ and $\mathrm{S}_{\mathrm{BET}}$ are the BET energy parameter and specific surface area assessed from the BET analysis of the $\mathrm{N}_{2}$ adsorption isotherms.

\begin{tabular}{cccccc}
\hline \hline Etching conditions & $<\mathrm{D}>(\mathrm{nm})$ & $\mathrm{D}_{\mathrm{O}}(\mathrm{nm})$ & $\mathrm{V}_{\mathrm{p}}(\mathrm{mL} / \mathrm{chip})$ & $\mathrm{C}_{\mathrm{BET}}$ & $\mathrm{S}_{\mathrm{BET}}\left(\mathrm{m}^{2} / \mathrm{chip}\right)$ \\
\hline Freshly etched & 7.6 & 7.2 & $4.4 .10^{-3}$ & 31 & 2.34 \\
\hline Oxidized & 7.2 & 6.6 & $3.6 .10^{-3}$ & 75 & 2.01 \\
\hline Oxidized and dipped in HF & 11.0 & 10.8 & $5.4 .10^{-3}$ & 43 & 1.73
\end{tabular}




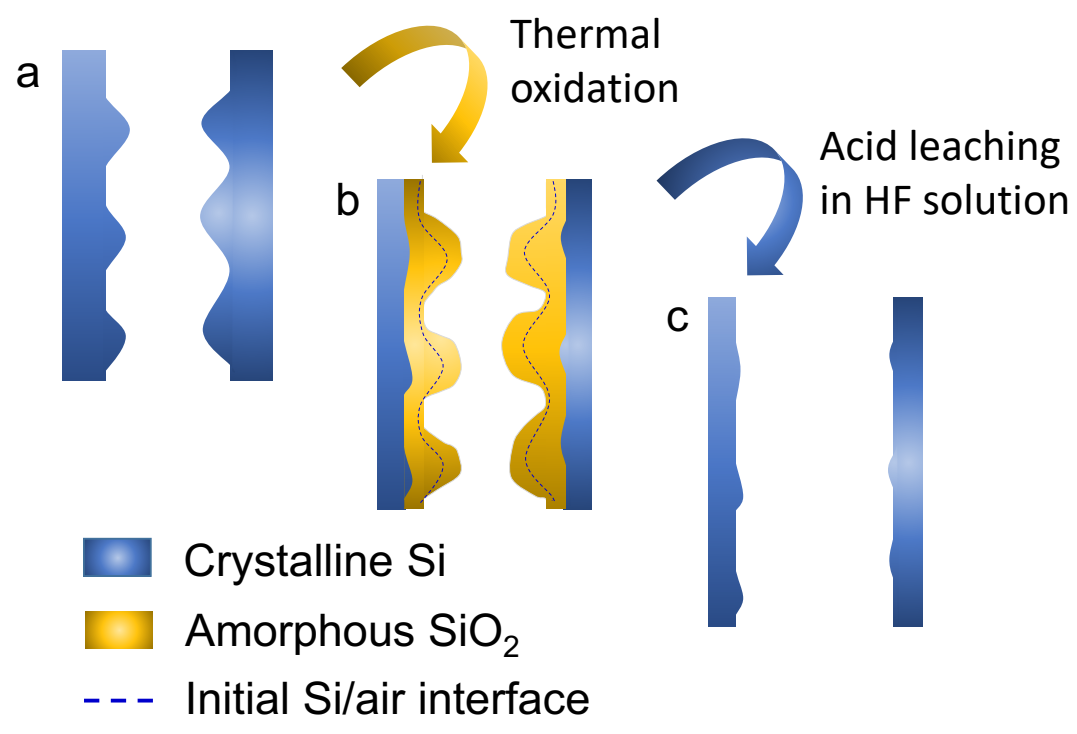

Figure 2. Schematic representation of the chemical pore smoothing process used to remove nanoscale features in the mesoporous Si. (a) freshly etched pSi, (b) pSi oxidized at $400^{\circ} \mathrm{C}$ for $1 \mathrm{~h}$, and (c) oxidized $\mathrm{pSi}$ at $400^{\circ} \mathrm{C}$ for $1 \mathrm{~h}$ after immersion in $\mathrm{HF}$ for $5 \mathrm{~s}$.

\subsubsection{Small angle Neutron (SANS) and x-rays (SAXS) scattering}

The different samples have been first measured in the dry state with SANS as reported in Figure 3a. The shape of the scattering curves is consistent with the geometry of the pores: the plateau and the oscillation at low Q are related to the pore diameter while the power law decrease of $\mathrm{I}(\mathrm{Q})$ at high $\mathrm{Q}$ is related to the surface morphology of the pores. The relative absolute intensities are also consistent with the schematic picture sketched in Figure 2. Despite the same neutron scattering contrast against air, the freshly etched pSi film (orange data) presents a higher level of intensity than the oxidized film after immersion in HF (blue data) because of a larger amount of scattering material. The oxidized pSi film, which possesses an additional contrast with air, leads to a larger scattering intensity (pink data). We put the freshly etched sample in different 
$\mathrm{H}_{2} \mathrm{O} / \mathrm{D}_{2} \mathrm{O}$ mixtures to determine the $\mathrm{H} / \mathrm{D}$ ratio that matches the silicon matrix density. ${ }^{22}$ The corresponding SANS curves are presented in the insert of Figure 3a; as expected, the contrast matched intensity induces a significant reduction of the scattering intensity level by a factor 10. Complete matching is almost reached for the oxidized pSi film after immersion in HF (blue data) for which the level of the scattering is very close to the instrument background $\left(\sim 10^{-}\right.$ $\left.{ }^{2} \mathrm{~cm}^{-1}\right)$. In contrast, for the freshly etched pSi sample that should be matched in principle, the matching is not efficient; this result might be due to the presence of some residual air bubbles inside the pores, as freshly etched silicon hydride terminated-pSi features an hydrophobic surface. On the contrary, the intensity level is still significant for the oxidized sample, which nicely illustrates the contribution of the oxidized layer. Indeed, the oscillation at intermediate $\mathrm{Q} \sim 0.1-0.2 \AA^{-1}$, which is already visible on the dry sample but more pronounced for the matched sample, is the signature of the shape of the oxidized layer. In order to reach higher Q values and probe the pore surface roughness to smaller lengthscales, we performed SAXS measurements on the same samples (Figure 3b). For all films, the Porod plot $\mathrm{I} \times \mathrm{Q}^{4}$ versus $\mathrm{Q}$ shows a plateau for the high $\mathrm{Q}$ values, which suggests that there are no significant changes of the film morphology following the HF treatment. This is in line with the electron microscopy observations, which indicate that the sample morphology remains overall similar. In addition, the local porosity is not modified - the $\mathrm{Q}^{-4}$ behavior is still visible - up to characteristic $\mathrm{Q}$ values close to $\sim 0.5-0.6 \AA^{-1}$ (corresponding to typical sizes $\sim 1.5-2 \AA$ ). These results suggest that surface roughness is on lengthscales larger than $\sim 1 \mathrm{~nm}$ as pores appear smooth on smaller lengths (Porod exponent $\sim 4$ ). Such a result is consistent with electron microscopy images which show that pores exhibit significant morphological disorder on such lengthscales $>1 \mathrm{~nm}$.

We can extract quantitative information about the oxidized layer using scattering considerations. Scattering from the freshly etched pSi film can be decomposed as the product 
of the structure factor with the form factor of the channel, i.e. $\mathrm{I}_{\text {freshly etched }} \sim \mathrm{S}_{\text {channel }} \mathrm{P}_{\text {channel. }}$. Considering the channel with an oxidized layer at its surface, the scattering intensity is proportional to the product of the structure factor and the form factor of the channel with the same product for the oxidized layer, i.e. $\mathrm{I}_{\text {oxidized }} \sim\left(\mathrm{S}_{\text {channel }} \mathrm{P}_{\text {channel }}\right) \times\left(\mathrm{S}_{\text {layer }} \mathrm{P}_{\text {layer }}\right)$. We can reasonably neglect the interactions between the oxidized layers of the different channel in the domain of high scattering vectors. Thus, the form factor of the oxidized layer can be extracted as $\mathrm{P}_{\text {layer }} \sim \mathrm{I}_{\text {oxidized }} /\left(\mathrm{S}_{\text {channel }} \mathrm{P}_{\text {channel }}\right) \sim \mathrm{I}_{\text {oxidized }} / \mathrm{I}_{\text {freshly etched. }} \mathrm{P}_{\text {layer }}$ as estimated from such a formula is shown in Fig. 3c; $P_{\text {layer }}$ displays a nice plateau as expected for an object with a finite size geometry. We have compared the resulting scattering function with the simple Guinier approximation that enables to calculate a typical size - a radius of gyration $R_{g}$ - without any geometrical assumptions. We find $\mathrm{R}_{\mathrm{g}}=1.2 \mathrm{~nm}$ (full purple line in Figure $3 \mathrm{c}$ ) which is fully consistent with the size of the oxidized layer usually found in the literature for pSi films oxidized upon such thermal conditions. ${ }^{23}$ Here we observe that the oxide layer of $\sim 1.2 \mathrm{~nm}$ is smaller than half of the wall thickness $(5-10 \mathrm{~nm})$ in the pSi samples of the present study, which further indicates that it is unlikely that the pores collapse or get perforated upon oxidation/removal of the oxide layer.
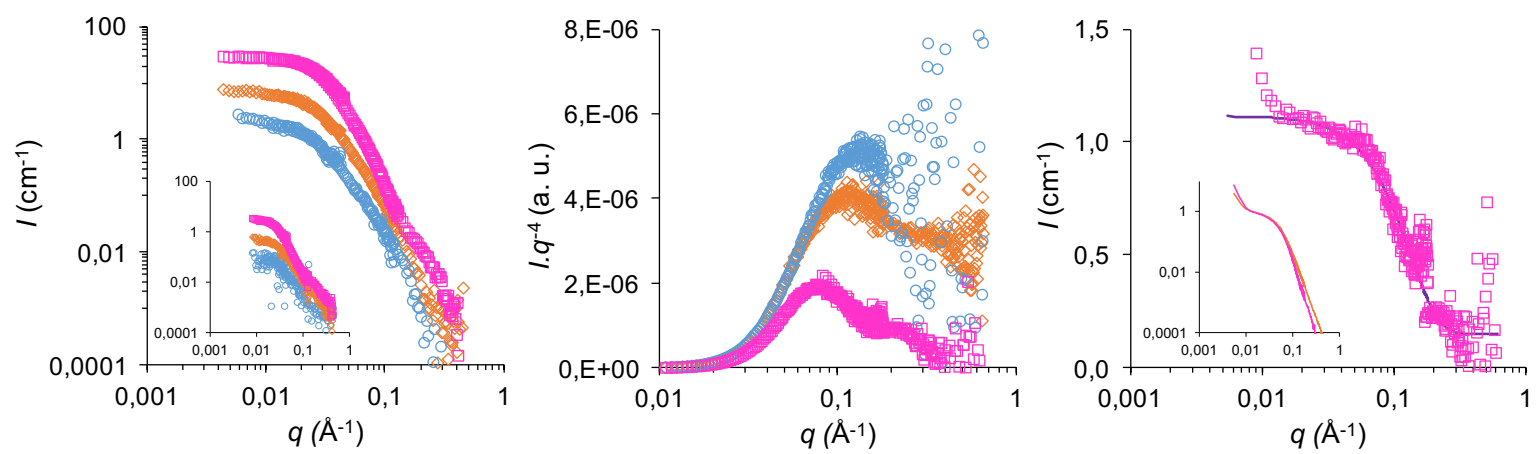
Figure 3. (a) SANS on dry samples: (४) freshly etched pSi sample, ( $($ ) pSi sample oxidized at $400^{\circ} \mathrm{C}$ for $1 \mathrm{~h},(\bullet) \mathrm{pSi}$ sample oxidized at $400^{\circ} \mathrm{C}$ for $1 \mathrm{~h}$ and immersed in a HF/ethanol solution for $5 \mathrm{~s}$. The insert shows SANS on the same samples immersed in a Si-contrasted matched solution, mixture of Ethanol/ $\mathrm{D}_{2} \mathrm{O}$ at $35.5 \%$ of $\mathrm{D}_{2} \mathrm{O}$ (vol.) (b) SAXS on the same dry samples. (c) Form factor of the oxide layer $\mathrm{P}_{\text {layer }}$ (see text) as obtained by dividing the scattered intensity for the oxidized pSi film (pink line on b) by that for the freshly etched pSi film (orange line on b), the full purple line is the result of the best fit against a Guinier function. The insert shows the same results plotted with a double logarithmic scale.

\subsection{Theory and molecular modeling}

\subsubsection{Lattice model of surface oxidation and oxide removal in porous silicon}

To investigate the changes in the surface area and porous volume of $\mathrm{pSi}$ upon oxidation and removal of the oxide layer in HF, we considered a simple lattice model. We started with a block of bulk silicon consisting of $73 \times 73 \times 161$ sites (Figure $4 a$ ). A site is a mesoscopic domain with a size from a few $\AA$ to a few $n m$. We then modeled pores of pSi by carving out of the block a cylindrical pore of a diameter $D$ closed at one end (Figure 4a). Surface roughness was introduced by modulating the pore diameter at a given position $k_{\mathrm{z}}$ along the $z$ axis and at an angle $\theta$ using a double cosine function ("egg box" roughness shown in Figure 4a):

$$
D\left(\theta, k_{\mathrm{z}}\right)=\operatorname{Max}\left(D_{0}, D_{0}+2 \Delta r_{\max } \cos \left(\lambda_{\mathrm{z}} k_{\mathrm{z}}\right) \cos \left(\lambda_{\theta} \theta\right)\right)
$$

where $D_{0}$ is the maximum pore diameter, $\Delta \mathrm{r}_{\max }$ is the maximum amplitude of the modulation along the $z$ and $\theta$ directions. $\lambda_{z}$ is the period of the modulation along the $z$ direction and $\lambda_{\theta}$ the period of the modulation along the $\theta$ direction. 
We verified that the qualitative conclusions drawn using the cosine function above remain valid when other types of modulation are used. At time $t=0$, all of the silicon domains are nonoxidized domains. After a timestep $\delta t$, the nearest domains $\mathbf{N}_{x}$ for each domain $\mathbf{N}$ are considered. If $\mathbf{N}_{\mathbf{x}}$ belongs to the pore void, surface oxidation is attempted: it consists of transforming both the non-oxidized domain $\mathbf{N}$ and the pore void domain $\mathbf{N}_{x}$ into oxide domains (Figure $4 b$ ). Such a surface oxidation step is based on the experimental fact that surface oxidation of silicon leads to a swelling of the oxidized region so that, at the end, the oxide layer thickness is about twice that of the initial non-oxidized silicon layer. ${ }^{24}$ If $\mathbf{N}_{\mathbf{x}}$ is already oxidized, volume oxidation is attempted: it consists of transforming the non-oxidized domain $\mathbf{N}$ into an oxide domain. Moreover, the closest void domain located at the same $k_{\mathrm{z}}$ position along the $z$ axis is also transformed into an oxidized domain in order to take into account the swelling of the matrix upon introduction of the oxygen atoms (Figure 4b). The physical picture behind this volume oxidation step is the diffusion of oxygen through oxidized domains to the oxide/non oxide interface. Oxidation of $\mathrm{pSi}$ is investigated by incrementing the time $\mathrm{t}+\delta t$ and repeating the procedure above. At each time step, the surface area and the porous volume of the oxidized sample and of the sample obtained after removal of the oxide layer can be estimated. The procedure is stopped when the surface and volume changes are equal to those observed experimentally.

The surface and volume oxidation steps are accepted with the same probability $P$. However, non oxidized domains having $n$ interfaces with the pore void undergo $n$ surface oxidation attempts. This assumption is physically reasonable as domains having a large surface area oxidize first as they offer easy access to oxygen present in the pore void. Such en edge effect can be rationalized in the framework of the Deal-Grove model. ${ }^{25}$ In this model, silicon oxidation is described as a 3-step process: (1) transport from the bulk gas phase to the outer surface of the 
oxidized material, (2) transport from the inner surface of the oxidized material to the $\mathrm{SiO}_{2} / \mathrm{Si}$ interface, and (3) incorporation of oxygen atoms into non oxidized $\mathrm{Si}$ to form $\mathrm{SiO}_{2}$. The third step is rate limiting in early oxide formation $(\sim \mathrm{nm}$, as expected in this work) since both gas transport and diffusion over very thin oxide layers are fast. Upon such conditions, all domains at a distance less than $\delta(t)$ from the gas/pSi interface are oxidized at a time $t$. Within this assumption, oxidation occurs over a larger depth $\left(\delta^{\prime}>\delta\right)$ on convex surfaces than flat and concave surfaces (see Figure S5 of the Supporting Information). Note that this argument, which supports formation of thicker oxides at convex surfaces, also holds when the second step is ratelimiting (i.e. diffusion through the oxide layer which is known to prevail for larger oxide films $>\mathrm{nm}$ ); indeed, the inner region of a convex surface is always easier to access (due to their large surface to volume ratio) than beneath a flat or concave region. Finally, in case the first step is rate-limiting (transfer from the bulk gas phase to the outer surface of $\mathrm{pSi}$ ), faster oxide formation at convex surfaces can be rationalized by the fact that the gas flux at sharp edges diverges. ${ }^{26}$ To account for such edge effects, which are linked to the local curvature $R$, the probability $P$ was multiplied by a factor $\left(\delta-R / R_{0}\right)^{1 / 3}$ (where $R_{0}=D_{0} / 2$ and $\left.\delta>1\right)$. This assumption was found to be essential to capture the concomitant decrease in the surface area with the increase in the porous volume upon oxidation and removal of the silicon oxide layer (note however that this simple model is not intended to provide a physical description of complex oxidation of rough pSi but to account for such edge effects in a phenomenological way). 
(a)

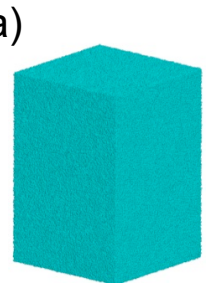

(b)

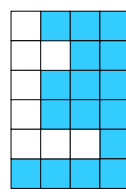

Pore void

Silicon

Oxidized

domain
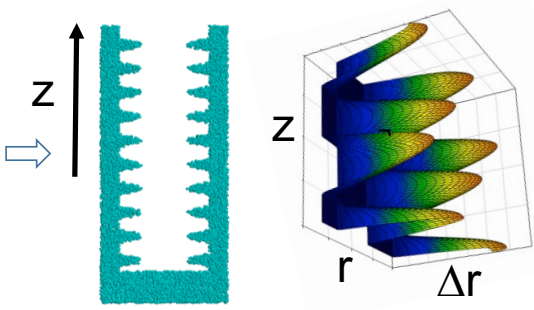

(c)

- Surface oxidation

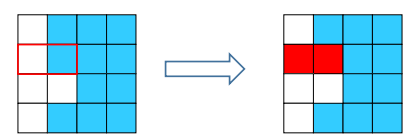

- Volume oxidation

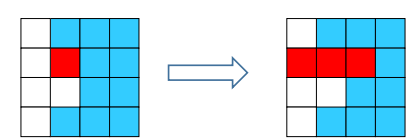

Figure 4. Representation of the on-lattice model used to simulate surface oxidation and oxide removal in porous silicon. (a) A lattice of $73 \times 73 \times 161$ domains is constructed. A cylindrical nanopore with a maximum pore diameter $D_{0}$ is carved out. Surface roughness $\Delta r$ is introduced by modulating the pore diameter using cosine functions. The right panel shows the surface roughness $\Delta r$ ("egg box" structure) used in the present work (see text). (b) Graphical representation of a small region of the surface of the inner pore wall of the pSi sample. Pore void are white squares, Si domains are cyan squares. At a time $t=0$, the algorithm starts from a non oxidized system where all matrix domains are silicon. (c) Graphical representation of the surface oxidation and of the volume oxidation. Pore voids are white squares, Si domains are cyan squares, oxide domains are red squares. If a square represents a Si domain that is adjacent to a pore void domain, surface oxidation is attempted in which both the Si domain and the pore void domain are transformed into oxide domains. If a domain has already been oxidized, volume oxidation is attempted in which both the closest non-oxidized domain and the closest void domain located at a position along the $z$ axis are transformed into oxide domains. 
In what follows, we consider a pore of diameter $D_{0}=54$ and a length $L=161$. The surface roughness was introduced using the cosine function described above with $\Delta z_{\max }=14, \lambda_{z}=0.45$, and $\lambda_{\theta}=6$. Figure 5a shows the evolution of the pSi sample prior to oxidation, after $n=5$ oxidation iterations, and after removal of the oxide layer. Figure S6 in the supporting information file also displays the radial density profiles for $\mathrm{pSi}$ prior to surface oxidation, for the formed silicon oxide layer, and after removal of the oxide layer. The formation of an oxide layer leads to a decrease in the pore radius from $R=26.5$ to $R=18.5$ as oxidation leads to swelling of the oxidized region, in good agreement with the experiments on the oxidation of the pSi surfaces. The pore radius here was estimated as the position of the air/silicon interface where the density is $\rho_{0} / 2$ with $\rho_{0}$ the density of bulk silicon. In contrast, upon removal of the oxide layer, the pore radius increases from $R=18.5$ to $R=28.2$. Interestingly, the decrease of the surface area after formation and removal of the oxide layer, as evidenced by the decrease of the width of the void/silicon interface (Figure S5 in the supporting information file), corresponds to a decrease of the surface roughness. The surface roughness in our model was estimated as the width $\Delta \xi$ of the silicon/void interface (again, defined as the region where the density increased from $\rho=0$ to $\rho_{0}$ ) normalized to the average pore radius $R: \Delta \xi / R$. The surface roughness $\Delta \xi / R$ was found to decrease from $66 \%$ down to $27 \%$ upon oxidation and removal of the oxide layer. Our model provides a physical picture of oxidation and surface roughness of real $\mathrm{pSi}$ as it describes in a qualitative way the concomitant increase in porous volume and decrease in surface area in correlation with the decrease of surface roughness. In particular, mapping of our lattice model onto the real samples considered in this work (see Table 1) suggests that pores with a diameter $D=7.6 \mathrm{~nm}$ and $D=9.5 \mathrm{~nm}$ should have initial surface roughness $\Delta \xi=2.5 \mathrm{~nm}$ and $\Delta \xi=3.2 \mathrm{~nm}$, respectively. Such surface disorder is large enough to affect capillary condensation and evaporation in nanoporous solids. ${ }^{12,23,24}$ Finally, our lattice 
model suggests that, after removal of the oxide layer, the surface roughness has decreased down to $\Delta \xi=1 \mathrm{~nm}$ and $\Delta \xi=1.3 \mathrm{~nm}$ for pores with diameter of $7.6 \mathrm{~nm}$ and $9.5 \mathrm{~nm}$, respectively.

\subsubsection{Molecular simulation of adsorption in oxidized and non-oxidized porous silicon}

To discuss the effect of surface oxidation and removal of the oxide layer on adsorption in $\mathrm{pSi}$, we performed Grand Canonical Monte Carlo (GCMC) simulations of nitrogen adsorption at 77 $\mathrm{K}$ in our model of oxidized and non-oxidized pSi. In this part, a lattice site was assumed to have a size of $0.225 \mathrm{~nm}$ so that pore sizes were consistent with those of the experimental samples. In so doing, the pores considered here have a mean pore size $D \sim 12 \mathrm{~nm}$ (freshly etched $\mathrm{pSi}$ ), $D \sim$ $8 \mathrm{~nm}$ (oxidized $\mathrm{pSi}$ ), and $D \sim 13 \mathrm{~nm}$ (pSi after removal of the oxide layer). Full details about the simulations can be found in the Supporting Information.

Figure $5 \mathrm{~b}$ shows the simulated $\mathrm{N}_{2}$ adsorption isotherms at $77 \mathrm{~K}$ in oxidized and non-oxidized pSi. We report the data for freshly etched porous silicon, oxidized porous silicon, and oxidized porous silicon after removal of the oxide layer. Adsorbed amounts have been normalized to the maximum adsorbed amount reached for the freshly etched porous silicon sample. We also show in Figure S7 of the supporting information file typical molecular configurations of nitrogen adsorbed at $77 \mathrm{~K}$ in each type of porous sample. The series of simulated adsorption isotherms captured all the features of the experimental adsorption isotherms.

(1) For all samples, adsorption starts at low pressure with the formation of a film adsorbed at the pore surface. As expected, preferential adsorption is observed in the cavities created by surface roughness at the pore surface. At a pressure much lower than the bulk saturating vapor pressure, $P_{0}$, capillary condensation in the pore occurs due to confinement. The condensation pressure increases with increasing the pore size, in agreement with the experimental data. 
(2) For all samples, the adsorption isotherm exhibits a hysteresis loop, which shows that the pore surface disorder and roughness are significant enough to delay capillary evaporation with respect to capillary condensation.

(3) Data for oxidized pSi show that both the adsorption and desorption branches are shifted towards lower pressure as the pore sizes become smaller upon oxidation. The shift of the desorption branch upon oxidation is slightly larger than for the adsorption branch, in agreement with the experimental data.

(4) Upon removal of the oxide layer, owing to the increase in pore size, the adsorption and desorption branches are located at larger pressures than those for the freshly etched and oxidized samples.

(5) The porous volume of the freshly etched sample, the oxidized sample, to the sample obtained after removal of the oxide layer was monitored from the maximum adsorbed amount. As expected, the volume change between each step is identical to that observed in the experiments. We determined the surface of each sample by applying the BET method to their corresponding adsorption isotherm. The $\mathrm{C}_{\mathrm{BET}}$ parameters for the non-oxidized porous silicon samples $\left(\mathrm{C}_{\mathrm{BET}} \sim 20-70\right)$ were smaller than what is usually observed for oxide surfaces $(80-120)$. In contrast, $\mathrm{C}_{\mathrm{BET}}$ for the oxidized sample, $\mathrm{C}_{\mathrm{BET}} \sim 100$, is close to what is expected for silicon oxide surfaces. 

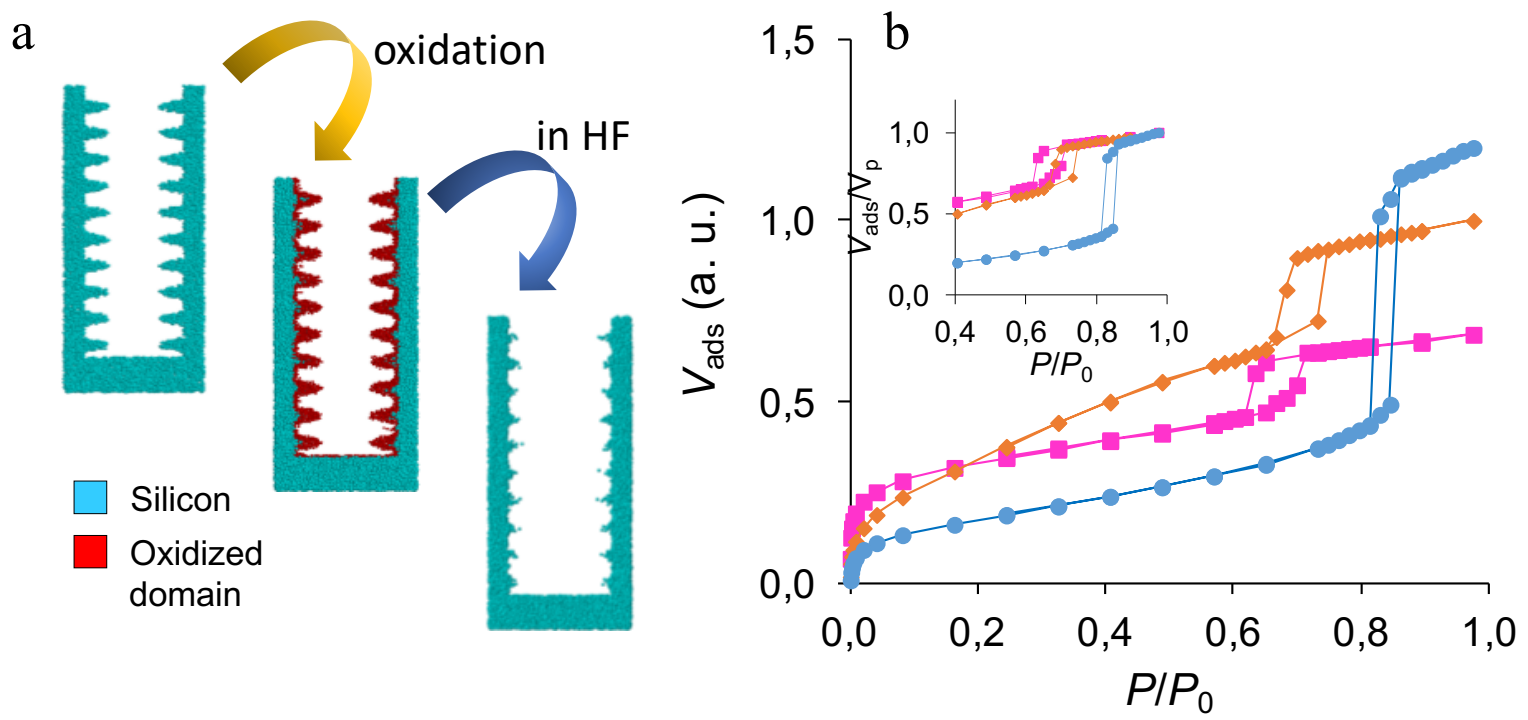

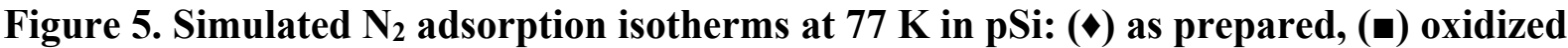
pSi, and $(\bullet)$ oxidized pSi after removal of the oxide layer. Pressures are in reduced unit with respect to the bulk saturating vapor pressure, $P_{0}$, for nitrogen at $77 \mathrm{~K}$. Adsorbed amounts have been normalized to the maximum adsorbed amount reached for the initial porous silicon sample. The insert shows the same data normalized to the maximum adsorbed amount for each sample $\left(V_{\text {ads }} / V_{\mathrm{p}}\right)$.

\section{Conclusion}

The rough internal pore surface in porous silicon can be smoothed by successive oxidation/oxide removal steps, therefore changing its physical properties as exemplified here with nitrogen adsorption. The process also increases the average pore diameter and pore volume. A combination of SEM images, small angle X-ray diffraction and neutron scattering measurements suggest that surface roughness is on a length scale of $>1 \mathrm{~nm}$ while the pores are smooth at smaller lengths. The data fit a simple coarse-grained model in which the finer scale features are oxidized and then removed from the surface of the pSi sample. Using such a coarse- 
grained model, Grand Canonical Monte Carlo simulations of nitrogen adsorption on freshly etched porous silicon, oxidized porous silicon, and porous silicon after selective removal of the oxide layer qualitatively agree with the experimental data, and support the interpretation that the pore surface is initially rough and that the oxidation/oxide removal procedure diminishes the surface roughness while increasing the pore diameter.

The results reported in this paper provide important insights into the surface properties of porous silicon which is a promising candidate for many applications ranging from optoelectronics, drug-delivery devices, sensors, etc. In particular, while previous works have reported evidence of the intrinsic surface disorder in porous silicon, the present results provide a quantitative estimate of such a surface roughness. Moreover, by providing a clear understanding of the mechanism by which oxidation and oxide removal decrease the surface roughness, the present work proposes a simple way to modify and control this important parameter. Such a simple procedure can be used to fine-tune the surface and textural properties of porous silicon.

Controlling the surface roughness in porous $\mathrm{Si}$ is believed to be of utmost importance for different applications. For instance, the microelectronic properties of porous $\mathrm{Si}$ such as its photoluminescence are known to be connected to the hydride surface groups at the surface of porous silicon and to the size of the nanocristallites. As a result, modulating in a controlled fashion the surface properties of porous Si can be a key asset in many applications where such electronic properties are involved. Moreover, a smoother internal surface might help to make more homogeneous coatings and/or surface functionalization. A larger porous volume could also improve the loading efficiency of drugs in drug delivery applications. Finally, the process presented here could also be used in sensing applications for better sensitivity. 


\section{Acknowledgments}

We thank Loïc Barré and Jalel M'Hamdi (IFPEN) for their help with the SAXS measurements.

We also thank Lydéric Bocquet, Laetitia Laversenne, Pierre Levitz, Renaud Denoyel and Daniel Brunel for stimulating discussions. This work was supported by the US National Science Foundation under Grant No. NSF DMR-1210417.

\section{References}

(1) Anglin, E.; Cheng, L.; Freeman, W.; Sailor, M. Adv. Drug Delivery Rev. 2008, 60, 12661277

(2) Torres-Costa, V.; Martin-Palma, R. J. Mater. Sci. 2010, 45, 2823-2838.

(3) Anglin, E.; Schwartz, M.; Ng, V.; Perelman, L.; Sailor, M. Langmuir. 2004, 11234-11269.

(4) Canham, L. (Editor) Handbook of Porous Silicon, Springer International Publishing, 2014

(5) Coasne, B.; Grosman, A.; Ortega, C.; Simon, M. Phys. Rev. Lett. 2002, 88.

(6) Wallacher, D.; Kunzner, N.; Kovalev, D.; Knorr, N.; Knorr, K. Phys. Rev. Lett. 2004, 92.

(7) Naumov, S.; Khokhlov, A.; Valiullin, R.; Karger, J.; Monson, P. Phys. Rev. E. 2008, 78.

(8) Casanova, F.; Chiang, C.; Ruminski, A.; Sailor, M.; Schuller, I. Langmuir. 2012, 28, 68326838.

(9) Cohan, L. J. Am. Chem. Soc. 1938, 60, 433-435.

(10) Grosman, A.; Ortega, C. Langmuir. 2011, 27, 2364-2374.

(11) Segal, E.; Perelman, L.; Cunin, F.; Di Renzo, F.; Devoisselle, J.M.; Li, Y.; Sailor, M. Adv. Funct. Mater. 2007, 17, 1153-1162.

(12) Coasne, B.; Galarneau, A.; Pellenq, R.J.M.; Di Renzo, F. Chem. Soc. Rev. 2013, 42, 4141.

(13) Schilling, J.; Birner, A.; Müller, F.; Wehrspohn, R.; Hillebrand, R.; Gösele, U.; Busch, K.; John, S.; Leonard, S.; Van Driel, H. Opt. Mater. 2001, 17, 7-10. 
(14) Lehman, V. Macroporous Silicon, in Electrochemistry of Silicon, Wiley-VCH Verlag GmbH, 2002, p.183-205.

(15) Trifonov, M.; Garín, A.; Rodríguez, L.;. Marsal, F.; Alcubilla, R. Phys. Status Solidi A. 2007, 204, 3237-3242.

(16) Ott, N., Nerding, M., Müller, G., Brendel, R. and Strunk, H. P. Phys. Status Solidi A. 2003, 197, 93-97.

(17) Müller, G., Nerding, M., Ott, N., Strunk, H. P. and Brendel, R. Phys. Status Solidi A. 2003, 197, 83-87.

(18) Müller, G.; Brendel, R. Phys. Status Solidi A. 2000, 182, 313

(19) Broekhof, JC.; De Boer, J. J. Catal. 1968, 10, 377.

(20) Aleksandrov, O.; Dusj, A. Semiconductors. 2008, 42, 1370-1376.

(21) Astrova, E. Handbook of Porous Silicon, L. Canham (Editor), Springer International Publishing, 2014, 589-598.

(22) Sailor, M.J., Porous silicon in Practice: Preparation, Characterization and Applications, Wiley-VCH, Weinheim, Germany, 2012.

(23) Pap, A.E.; Kordas, K.; Toth, G.; Levoska, J.; Uusimaki, A.; Vahakangas, J.; Leppavuori, S.; George, T.F. Appl. Phys. Lett. 2005, 86, 041501.

(24) Gommes, C. Langmuir. 2012, 28, 5101-5115.

(25) Deal, B. E.; Grove; A. S. J. Appl. Phys. 1965, 36, 3770-3778.

(26) Bocquet, L. Am. J. Phys. 2007, 75, 148. 